\title{
Temperature dependence of protein dynamics simulated with three different water models
}

Article

Accepted Version

Glass, D. C., Krishnan, M., Nutt, D. R. and Smith, J. C. (2010) Temperature dependence of protein dynamics simulated with three different water models. Journal of Chemical Theory and Computation, 6 (4). pp. 1390-1400. ISSN 1549-9618 doi: https://doi.org/10.1021/ct9006508 Available at https://centaur.reading.ac.uk/18176/

It is advisable to refer to the publisher's version if you intend to cite from the work. See Guidance on citing.

To link to this article DOI: http://dx.doi.org/10.1021/ct9006508

Publisher: American Chemical Society

All outputs in CentAUR are protected by Intellectual Property Rights law, including copyright law. Copyright and IPR is retained by the creators or other copyright holders. Terms and conditions for use of this material are defined in the End User Agreement.

www.reading.ac.uk/centaur 
Central Archive at the University of Reading

Reading's research outputs online 


\title{
Temperature dependence of protein dynamics simulated with
}

\author{
various water models
}

\author{
Dennis C. Glass ${ }^{1}$, M. Krishnan ${ }^{1}$, David R. Nutt ${ }^{2}$, and Jeremy C. Smith ${ }^{1}$ \\ ${ }^{1}$ University of Tennessee/ORNL Center for Molecular Biophysics \\ Oak Ridge National Laboratory, Oak Ridge, Tennessee \\ ${ }^{2}$ The University of Reading, Reading, UK
}

\begin{abstract}
The effect of variation of the water model on temperature dependent protein and hydration water dynamics is examined by performing molecular dynamics simulations of myoglobin with the TIP3P, TIP4P, and TIP5P water models and the CHARMM protein force field at temperatures between 20 and $300 \mathrm{~K}$. The atomic mean-square displacements, solvent reorientational relaxation times, pair angular correlations between surface water molecules and time-averaged structures of the protein are all found to be similar and the protein dynamical transition is described almost indistinguishably for the three water potentials.
\end{abstract}


The results provide further evidence that variation of the water model without loss of accuracy can be considered, opening up the possibility of choosing the water model employed in protein simulations according to the properties to be investigated.

\section{Introduction}

Water plays an important role in many chemical and biological processes $[1,2,3$, $4,5,6,7,8,9,10]$. For example, hydration water strongly influences the threedimensional structure, dynamics, and function of proteins [2]. Water-protein interactions modify the free energy landscape that determines the folding, structure and stability of proteins $[3,4,5,6]$. Internal protein dynamics, which are required for biological function, are dependent on the level of hydration [7] and dynamical coupling between the protein and water influences the conformational flexibility of proteins $[8,9,10,11]$.

Protein hydration water can be grouped into two classes: internal water molecules which can play structural and/or catalytic roles $[12,13]$, and external surface water molecules. Hydration water is experimentally estimated to account for $10-15 \%$ of the total cell water $[14,15]$ of which a small fraction of $\approx 0.1 \%$ is internal water molecules. Properties of water in the external hydration shell are modified compared to the bulk, with, for example, changes in av- 
erage density $[16,17,18]$ and perturbations in translational and rotational dynamics, and these changes have been extensively investigated using techniques such as neutron scattering $[14,19,20,21,22,23,24,25,26]$, nuclear magnetic resonance [27, 28, 29, 30, 31, 32], fluorescence spectroscopy [33], midinfrared pump-probe spectroscopy [34], and molecular dynamics (MD) simulations $[22,35,36,37,38,39,40,41]$.

Empirical, molecular mechanics force fields, such as CHARMM [42, 43, 44], AMBER [45], GROMOS [46] and OPLS-AA [47] are widely employed in atomistic MD simulations of biological molecules and, in order to represent the proteinsolvent energy accurately, most molecular simulation applications employ explicit water models. A large number of water models is available. However, individual biomolecular force fields have normally been parametrized for use with a single water model (see e. g. [48]) such that, during the parameterisation, care can be taken to correctly balance water-water, water-protein, and protein-protein interactions. Nethertheless, the question arises as to whether the water:protein potentials are sufficiently robust that alternative water models may be employed with any given biomolecular force field without serious loss of accuracy. Flexibility in the choice of the water model may be of particular interest when the system is to be simulated at nonphysiological temperatures or pressures, under which circumstances different water models may exhibit significantly different properties (see e.g. [49]), 
and/or when water properties are specifically under investigation in which case a detailed water potential may be appropriate.

In recent work, effects of varying the water model in molecular mechanics and dynamics calculations on the hydration of N-methylacetamide (NMA) hydration and other small solute molecules, and a small protein using the CHARMM force field [42, 43, 44] were examined [50]. The overall description of solvation and biomolecular properties were found to be similar for the three models tested: TIP3P, TIP4P, and TIP5P $[49,51]$. The CHARMM protein force field was parameterized for use with the TIP3P potential. However, the results provide an indication that molecular simulations with the CHARMM force field may in some cases be performed with water models other than TIP3P.

In the present work, we focus on the effect of varying water models on the temperature dependence of internal protein motion. Experimental and theoretical studies have found that proteins undergo a transition in internal dynamics at $T_{g} \approx 180-220 K[52,53,54,55,56,57,58,59,60,61,62,63]$, characterized by a rapid increase in the average protein atomic mean-square displacement above $T_{g}$. An additional low-temperature transition $(\sim 150 \mathrm{~K})$, that has been attributed to the activation of methyl group rotations, is present also in dehydrated proteins $[64,65,66,67,68,69]$, whereas the transition at $T_{g}$ is strongly coupled to the solvent dynamics $[2,60,61,62,68,70,71,72,73,74,75,76,77,78,79]$. The dynami- 
cal transition at $T_{g}$ can be eliminated when dehydrating the protein or coating it with bioprotectant [80] and the value of $T_{g}$ can be shifted by changing the solvent composition [81]. MD simulations have demonstrated that the $T_{g}$ dynamical transition is driven by translational solvent dynamics $[61,82]$. For some proteins, the $T_{g}$ dynamical transition has also been correlated with protein activity [57, 59, 71, 83, 84] although activity has also been observed for $T<T_{g}[85,86,87,88]$.

The question arises as to whether the dynamical properties of the protein are affected by the choice of the water model used. To investigate this, we have performed simulations of myoglobin at temperatures ranges from $20 \mathrm{~K}$ to $300 \mathrm{~K}$ using the TIP3P, TIP4P, or TIP5P potentials and the CHARMM protein force field $[42,43,44]$. The protein dynamical transition was found to be unaffected by changes in the solvent model. Moreover, although the bulk properties of the three water models are markedly different, when interacting with the protein surface, the three water models behave similarly.

\section{Methods}

The set of models considered here is the TIP3P, TIP4P, and TIP5P family $[49,51]$. TIP3P is the standard model in the widely-used CHARMM force fields. However, TIP4P and TIP5P are easy to implement for use with CHARMM and exhibit improved water properties, as described below. 
The TIP geometries and associated parameters are shown in Fig. 1 and Tab. 1. In the TIP3P model, charges are placed at the hydrogen positions and on the oxygen, resulting in three interaction sites. A van der Waals term provides additional nonbonding interactions involving the oxygen atoms only [51]. Geometrical parameters were determined according to experimental gas phase values.

TIP3Pm is a slightly modified version of TIP3P, commonly used with CHARMM [42, 43, 44], and including additional van der Waals interaction sites at the hydrogen positions $[89,90]$. The effect of these additional terms on the properties of TIP3P has been shown to be small [91]. Therefore, in the following, TIP3P is used to refer to the CHARMM-modified version, TIP3Pm.

The combination of TIP3P with the CHARMM force field has proven to be useful for examining the structure and dynamics of biomolecular systems. However, although TIP3P adequately describes the first hydration shell of bulk water, it lacks accuracy for the second hydration shell, for which the corresponding peak in the oxygen-oxygen radial distribution function is almost completely absent $[49,90]$. TIP4P, in which the oxygen charge site is moved along the $\mathrm{HOH}$ bisector towards the molecular center of mass, better reproduces experimental distribution functions than TIP3P [51]. In TIP5P, there are two lone-pair interaction sites, L, moved from the oxygen along the $\mathrm{HOH}$ bisector away from the hydrogens and symmetrically placed out of the $\mathrm{HOH}$ plane with $\angle_{L O L}=109.47^{\circ}$ [49]. The TIP4P and TIP5P 
oxygens carry no charge. TIP5P, explicitly incorporating tetrahedrality in the water model, is especially successful in reproducing water density over a wide range of temperatures [49].

Here, the CHARMM program package version $\mathrm{c} 33 \mathrm{~b} 2$ was used to perform MD simulations of hydrated myoglobin with the CHARMM22 force field and TIP3P, TIP4P, or TIP5P water [42, 43, 44]. The $1.15 \AA$ A-resolution myoglobin structure 1A6G, taken from the RCSB Protein Data Bank (www.pdb.org) [92], was used as the starting protein configuration. The model was constructed as in Ref. [61] to mimic a hydrated powder sample and thus model the experimental neutron scattering setup of Ref. [75], the data from which serve as a reference here. To do this, the protein was solvated by placing it in a box of water, retaining only those 492 molecules closest to the protein. The temperature dependent dynamical properties in the present simulations were found to be very similar to those derived from NPT simulations in solution on the same myoglobin structure [69].

Electrostatic interactions were truncated using a shift function with a $12 \AA$ cutoff, and a switch function was used for truncation of van der Waals interactions between 10 and $12 \AA$. The SHAKE algorithm was used to constrain all bond lengths involving hydrogens [93]. The structures were energy minimized using 600 steepest descent steps and 2500 conjugate gradient steps with the protein atoms fixed, then with fixed solvent allowing the protein to move, and finally without any constraints. 
After heating to the desired temperature in steps of $5 \mathrm{~K}$ every 1000 dynamics steps, the system was equilibrated for 400 ps. Subsequently, 1 ns production runs were performed in the NVT ensemble at temperatures from 20 to $300 \mathrm{~K}$ in intervals of $20 \mathrm{~K}$ and in smaller intervals of $10 \mathrm{~K}$ between 120 and $240 \mathrm{~K}$. The system was kept at constant temperature using the weak coupling algorithm of Ref. [94]. A time step of $1 \mathrm{fs}$ was used for the integration of the equations of motion. Coordinates and velocities were saved every 50 steps. The simulation protocol was the same for all temperatures and water models.

In order to examine in more detail the influence of the water model on the time averaged protein structure at $300 \mathrm{~K}$, ten additional simulations of $1 \mathrm{~ns}$ length were performed for each water model, starting with different, randomly-chosen, initial velocity assignments.

To avoid potential artifacts no restraining potential was applied to the water molecules to prevent evaporation. Subsequently for $T>260 K$, a small number of molecules evaporated from the water shell surrounding the protein and these were excluded from all analyses. No evaporation occured for $T<260 K$, including during extended 7 ns simulations with TIP3P at 180, 220, and $260 \mathrm{~K}$.

In addition to the above calculations, for comparison purposes simulations of bulk water using the TIP water models were performed. For these, GROMACS 4.0 [95] was used to generate a $30 \AA$ cubic box at physiological conditions containing 
895 molecules for TIP3P, 886 for TIP4P, and 878 for TIP5P. The electrostatic interactions were treated in the same way as for the above protein simulations, and periodic boundary conditions were applied. The configurations were energy minimized using 600 steepest descent steps, equilibrated for 500 ps at the desired temperatures and, subsequently, $1 \mathrm{~ns}$ production runs were performed in the NVT ensemble at temperatures from 20 to $300 \mathrm{~K}$ in intervals of $20 \mathrm{~K}$ and in smaller intervals of 10 $\mathrm{K}$ between 100 and $260 \mathrm{~K}$. The system was kept at constant temperature using the weak coupling algorithm of Ref. [94]. A time step of 2 fs was used. Coordinates were saved every 50 steps. The simulation protocol was again the same for all temperatures and water models.

\section{Results}

\subsection{Time-averaged structures}

The influence of the water models on the average structure of myoglobin is first investigated. The time-averaged structures were calculated for all 30 independent simulations at $300 \mathrm{~K}$ and were compared using a root mean square deviation (RMSD) per residue, defined here as:

$$
R M S D_{i}^{A, B}=\sqrt{\frac{\sum_{j=1}^{N_{i}}\left(x_{j}^{A}-x_{j}^{B}\right)^{2}}{N_{i}}}
$$


where $i$ is the residue number, $N_{i}$ is the number of protein heavy atoms in residue $i, A$ and $B$ are any two given time-averaged structures, and $x_{j}^{A / B}$ denotes the heavy atom coordinates. Convergence was checked by comparing RMSD values from the first and second half of each simulation, which were found to be in close agreement.

Fig. 2 shows the backbone heavy-atom RMSD per residue based on the average over all pairs $(A, B)$ of structures solvated in the same or in different water models. "SELF" refers to RMSD values between protein structures solvated by the same water model whereas "CROSS" refers to deviations in configurations between the models, for example, structures of the protein in TIP3P compared with structures in TIP4P or TIP5P.

Neglecting the five C- and N-terminal residues, the $R M S D_{i}^{A, B}$, averaged over residues is $0.47 \pm 0.25 \AA$, averaged over "SELF" is $0.46 \pm 0.26 \AA$, averaged over "CROSS" is $0.49 \pm 0.25 \AA$, and the average difference between "SELF" and "CROSS" is $0.035 \pm 0.026 \AA$. Therefore, the RMSD per residue is similar for both the "SELF" and "CROSS" data sets, indicating that variation of the water model does not significantly influence the time-averaged protein RMSD. 


\subsection{Mean-square displacement}

The mean-square displacement $\left\langle r^{2}(t)\right\rangle$ is defined as:

$$
\left\langle r^{2}(t)\right\rangle=\left\langle\frac{1}{N} \sum_{i=0}^{N}\left(r_{i}\left(t+t_{0}\right)-r_{i}\left(t_{0}\right)\right)^{2}\right\rangle_{i, t_{0}}
$$

where $N$ is the number of atoms, $\left(r_{i}\left(t+t_{0}\right)-r_{i}\left(t_{0}\right)\right)$ is the displacement of atom $i$ in time $t$, and $\langle\cdot\rangle_{i, t_{0}}$ represents the ensemble average, approximated as a time average over $t_{0}$ by assuming ergodicity.

The mean-square displacement averaged over the atoms of myoglobin was calculated for all temperatures and water models and the results are shown in Fig. 3 A. The inset shows an expanded view of the low-temperature region, up to $T \approx 210 K$. The MSD rises linearly for low temperatures until a first change in the gradient at $T \approx 150 \mathrm{~K}$, which has been attributed to the activation of methyl group rotations $[66,68,69]$. At $T \approx 220 K,\left\langle r^{2}(t)\right\rangle$ exhibits a further increase in the gradient corresponding to the solvent-driven dynamical transition. $\left\langle r^{2}(t)\right\rangle$, both for $<220 \mathrm{~K}$ and $>220 \mathrm{~K}$, is similar using all three TIP water models.

Fig. $3 \mathrm{~B}$ shows $\left\langle r^{2}(t)\right\rangle$ of the water molecules in the protein simulations as a function of temperature. The insets B.1 and B.2 give expanded views since the data cover multiple orders of magnitude. The profiles qualitatively resemble Fig. 3 A, i.e., the MSD for the water molecules rises linearly for low temperatures until, interestingly, a first change in slope is seen at $T \approx 150 K$ (Fig. 3 B.2) followed by a 
second transition at $T \approx 200 K$ (Fig. 3 B.1).

The $\sim 220 \mathrm{~K}$ transition in the protein has been previously observed and the strong coupling between the solvent and protein characterized $[55,58,61,71,74,76,78$, $82,96,97,98,99,100,101,102]$. However, the low-T, $150 \mathrm{~K}$ change in slope for water was unexpected, and the question therefore arises as to whether it would occur independent of the protein. To check this, a set of simulations of pure TIP water was performed, as described in Methods. Fig. 4 A shows the TIP water mean-square displacements as a function of temperature. Comparison between Fig. 3 B and 4 A indicates that, while the dynamical transition behaviour of the protein and protein hydration water are similar, they do not resemble that of the bulk water models. The data for the three TIP models exhibit similar properties in that $\left\langle r^{2}(t)\right\rangle$ is linear for $T \leq T_{l}$. However unlike in the solution simulations, the temperature at which the slope changes, $T_{l}$, is markedly different for the three models with $T_{l} \approx 110 \mathrm{~K}$ for TIP3P, $130 \mathrm{~K}$ for TIP4P, and $190 \mathrm{~K}$ for TIP5P. Further, again unlike the data for the hydrated protein, the bulk data do not show two distinct changes in slope.

The self-diffusion constant, D, was calculated from the linear part of each meansquared displacement, at i.e. $t>200$ ps (see Fig. 4 (B)), using the Einstein relation:

$$
\lim _{t \rightarrow \infty}\left\langle\left|r\left(t_{0}+t\right)-r\left(t_{0}\right)\right|^{2}\right\rangle=6 D t
$$

where $r(t)$ is the position of the water oxygen atom, and $\langle\cdot\rangle$ denotes averaging over 
both time origins $t_{0}$ and the water molecules. $\mathrm{D}$, in units of $10^{-5} \mathrm{~cm}^{2} / \mathrm{s}$, at $300 \mathrm{~K}$ is found to be $5.46 \pm 0.02$ (TIP3P), $3.60 \pm 0.06$ (TIP4P), and $2.82 \pm 0.02$ (TIP5P). Corresponding values in the literature vary depending on the simulation setup, but reported values using a similar protocol to that used here (i.e. shift electrostatics) in the NPT ensemble are $5.8 \pm 0.2$ (TIP3P), $3.78 \pm 0.02$ (TIP4P), $2.94 \pm 0.06$ (TIP5P) [103] in good agreement with the present work. For completeness, in Table 2, D is also given for the other temperatures.

\subsection{Heterogeneous distribution of anharmonic motions among protein residues.}

To quantify the fraction of protein residues that exhibit large anharmonic dynamics, the MSD per residue $\left(\left\langle r^{2}(t)\right\rangle_{i}\right.$, where $i$ denotes the residue index) was calculated. $\left\langle r^{2}(t)\right\rangle_{i}$ is decomposed into harmonic and anharmonic components as follows:

$$
\left\langle r^{2}(t)\right\rangle_{i}=\left\langle r^{2}(t)\right\rangle_{i, \text { harmonic }}+\left\langle r^{2}(t)\right\rangle_{i, \text { anharmonic }}
$$

where $\left\langle r^{2}(t)\right\rangle_{i, \text { harmonic }}$ is obtained by linearly fitting $\left\langle r^{2}(t)\right\rangle_{i}$ for $T \leq 140 K$ and extrapolating to higher $\mathrm{T}$.

A residue is denoted as exhibiting anharmonic dynamics if $\left\langle r^{2}(t)\right\rangle_{i, \text { anharmonic }}>$ $\left(\left\langle r^{2}(t)\right\rangle_{i, \text { harmonic }}+2 \sigma\right)$, where $\sigma$ is the standard deviation at the onset of anharmonicity $(140 \mathrm{~K})$. As a control, it was determined whether the onset of anharmonic- 
ity depends on the temperature interval chosen for the estimation of harmonic dynamics: using $20-100 \mathrm{~K}$ as the fitting interval and $\sigma_{100 K}$, the procedure was found to give similar results.

Fig. 5 shows the temperature dependence of the fraction of residues exhibiting anharmonic dynamics. For $T \leq 140 K$, almost all residues exhibit only harmonic motion, with $\left\langle r^{2}(t)\right\rangle_{i}$ similar to each other, while an abrupt change is evident at higher $\mathrm{T}$, as an increasing fraction of residues exhibits anharmonic dynamics. Even well above the dynamical transition temperature at $260 \mathrm{~K}, \sim 25 \%$ of the residues still remain harmonic. This result is consistent with previous simulation work on myoglobin, in which the onset of anharmonicity was found to be gradual with $\mathrm{T}$ [62]. Again, when comparing the protein simulations using different water models, there are no statistically significant differences.

\subsection{Reorientational relaxation time}

The rotational dynamics of water can be characterized by the water dipole orientation autocorrelation function, $C(t)$ :

$$
C(t)=\left\langle\vec{e}_{i}\left(t+t_{0}\right) \cdot \vec{e}_{i}\left(t_{0}\right)\right\rangle_{i, t_{0}}
$$

where $\vec{e}_{i}(t)$ is a unit vector along the water dipole. For liquids, $C(t)$ decays to zero as the dipole loses its memory of its initial orientation. 
Since the structural and dynamic properties of the hydration layer water molecules depend on the heterogeneous surface roughness and charge distribution $[22,25,40,41,104]$, a multi-exponential decay is expected. From the protein simulations, the reorientational relaxation time of water, $\tau$, was calculated by fitting the following triple exponential function to $C(t)$ :

$$
\begin{aligned}
C(t)= & a_{0} \exp \left(-t / \tau_{0}\right)+a_{1} \exp \left(-t / \tau_{1}\right) \\
& +\left(1-a_{0}-a_{1}\right) \exp \left(-t / \tau_{2}\right)
\end{aligned}
$$

Eq. 6 was found to capture the decay in the target data, whereas the fitting procedure failed or gave worse results for simpler fitting functions. The relaxation time, $\tau$, was derived using the following relation:

$$
\tau=a_{0} \tau_{0}+a_{1} \tau_{1}+\left(1-a_{0}-a_{1}\right) \tau_{2}
$$

Fig. 6 A shows $C(t)$ together with the fit of Eq. 6 for TIP3P hydration water at different temperatures. $C(t)$ for TIP4P and TIP5P hydration water exhibit similar decay behaviours (not shown). In general, the profiles consist of a fast decay on the picosecond timescale followed by slower dynamics. For most temperatures, $C(t)$ does not fully decay to zero on the present timescale of $\sim 500$ ps. For temperatures $T>220 K$ (above the dynamical transition temperature) $C(t)$ decays rapidly $(<50$ 
ps) to $\sim 0.5$, but the decay is much slower at lower $\mathrm{T}$.

There is good agreement between the fitted curves of Eq. 6 and the data (Fig. 6 A). Fig. 7 and Tbl. 4 present the temperature dependence of the resulting fitted parameters. At low $\mathrm{T}(\leq 180 K)$, the process associated with relaxation time $\tau_{2}$ dominates, with a weight factor, $1-a_{0}-a_{1} \approx 1$. A decrease of $1-a_{0}-a_{1}$ (i.e., an increase of $a_{0}$ and/or $a_{1}$ ) is observed for $T>180 K$, indicating the activation of additional relaxation processes. For $T \geq 240 K$ the three components are approximately equally weighted.

The temperature dependences of the weights and values of $\tau_{0}$ and $\tau_{1}$ behave very similarly to each other, with the weight of the $\tau_{1}$ component being larger and the values being $\sim 10$ times larger than $\tau_{0} . \tau_{0}$ and $\tau_{1}$ have broad maxima at $\sim 260 \mathrm{~K}$ and $210 \mathrm{~K}$, respectively. For temperatures $T \leq 180 K$, the $\tau_{1}$ component jumps to high values, accompanied by a drop in its weight.

The relaxation times $\tau$, derived by Eq. 7, are given in Fig. 6 B. Relaxation times at $300 \mathrm{~K}$ from the full sets of simulations at this temperature are $31 \pm 3,35 \pm 3$, and $32 \pm 6$ ps (TIP3P, TIP4P, and TIP5P) and rise by two orders of magnitude as the temperature decreases to $160 \mathrm{~K}$.

At temperatures $T>T^{*}$, glass-forming liquids exhibit an Arrhenius relaxation mechanism $\tau \propto \exp \left(-E_{\beta} / k_{B} T\right)$ due to the behaviour of the $\beta$-relaxation which, at $T^{*}$, splits into a fast $\beta$-relaxation and a slow $\alpha$-relaxation $[105,106]$. The $\alpha$ - 
relaxation may often be described with a Vogel-Fulcher-Tammann (VFT) equation over the range $T_{g}<T<T^{*}$, i.e.,

$$
\tau(T)=\tau_{c} \exp \left(\frac{A}{T-T_{0}}\right)
$$

with fitting parameters $\tau_{c}, A$, and $T_{0}$. Cooperativity of $\beta$-relaxation events has been suggested as the origin of the $\alpha$ relaxation. A similar VFT relationship between $\tau$ and $\mathrm{T}$ has been used in glass physics [105], where $T_{0}$ has been hypothesized

to be the Kauzmann temperature and $A=\frac{E_{\beta}}{R} \frac{T^{*}-T_{0}}{T^{*}}$ with $E_{\beta}$ as the activation energy for the $\beta$-relaxations. The Kauzmann temperature is the temperature, at which the entropies of the supercooled liquid and the corresponding crystal are in principle be equal [106, 107].

Angell proposed a "fragile-to-strong" classification of liquids in which relaxation times of "strong" liquids follow an Arrhenius trend (e.g. $\mathrm{SiO}_{2}$ ) whereas "fragile" liquids deviate from such a behaviour [108]. The VFT relation describes well the present data for $T>160 K$, thus classifying the protein hydration shell water as a "fragile" liquid in Angell's scheme.

\subsection{Local orientational ordering}

The local orientational ordering of water dipoles can be quantified by the distancedependent Kirkwood $G$-factor, defined as follows [109]: 


$$
G_{K, h}(r)=\left\langle\vec{\mu}_{i} \cdot \vec{M}(r)\right\rangle
$$

with

$$
\vec{M}(r)=\sum_{r_{i j} \leq r} \vec{\mu}_{j}
$$

where $\vec{\mu}_{i}$ denotes a unit vector in the direction of the dipole moment of molecule i. $G_{K, h}(r)$ is equal to two if a pair of water dipoles is parallel. Elevated $G_{K, h}(r)$ therefore corresponds to high angular correlations between water molecules.

Fig. 8 shows $G_{K, h}^{r_{\max }}(T)$, as a function of temperature for different water models, where $r_{\max }$ is the most-probable near neighbour distance, taken to be equal to the position of the first peak in the water oxygen-oxygen radial distribution function $g_{O O}(r)(\sim 2.8 \AA)$. Other simulation studies obtained similar values for $G_{K, h}^{r_{\max }}(T)$ at $\sim 2.8 \AA$ and $300 \mathrm{~K}[110,111]$.

A systematic decrease in $G_{K, h}^{r_{\max }}(T)$ is evident above $210 \mathrm{~K}$ for all water models, associated with the increased diffusion (see Fig. 3 B). TIP4P exhibits lower angular correlation in the hydration layer, indicating that the TIP4P dipoles are less strongly aligned. The results reported in Figs. 3 A/B and Fig. 8 are consistent with dynamical coupling between the protein and the solvent since the transition consistently occurs at $\sim 220 \mathrm{~K}$, captured by all TIP water models investigated here. 


\section{Conclusions}

The effect of the variation of the water model on the temperature dependence of protein and hydration water dynamics have been investigated here by performing molecular dynamics simulations of hydrated myoglobin. Both protein and water properties were analysed, including the time-averaged structures of the protein, the average mean-square displacements of the protein and water atoms, the solvent reorientational relaxation times, and pair angular correlations between the water molecules.

Variation of the water model between TIP3P, 4P, and 5P leads to the same timeaveraged structures of the protein to within statistical error. Also, for all three water models, the temperature-dependent mean-square displacement exhibits the wellknown dynamical transition at $T \approx 220 K$.

Mid-infrared pump-probe spectroscopy on the dynamics of $\mathrm{HDO}$ in $\mathrm{H}_{2} \mathrm{O}$ yielded an orientational lifetime of $\tau_{P P}=2.5 \mathrm{ps}$ for bulk water and $\tau_{P P}>10 \mathrm{ps}$ for "immobilized" water in the solvation shell of tetramethylurea [34, 112] which corresponds to a retardation factor of 4 . The bulk water relaxation time $\tau_{D}$ was derived to be 8.8 ps from molecular dynamics studies and 7 ps from experiments on dielectric relaxation, both at $303 \mathrm{~K}[113] .^{1}$

\footnotetext{
${ }^{1}$ Pump-probe experiments measure different lifetimes than dielectric relaxation experiments or the present simulation data: whereas the lifetime $\tau_{P P}$ from pump-probe experiments is related to the second-order correlation function $\left\langle P_{2}(\cos \theta(t))\right\rangle$ (where $P_{2}$ is the second-order Legendre polynomial), the lifetimes $\tau_{D}$ from dielectric relaxation and the present work are determined by the first-order correlation function $\left\langle P_{1}(\cos \theta(t))\right\rangle$. The ratio between $\tau_{P P}$ and $\tau_{D}$ depends on the details of molecular diffusion: $\tau_{P P}=3 \tau_{D}$ for simple rotational diffusion, but is different for other types of dynamics [114].
} 
Experiments and simulations have shown that the reorientational dynamics of protein hydration water is slowed down relative to the bulk e.g., $[6,27,28,35,37$, 41], with the slowing down being influenced by heterogeneous surface roughness and charge distribution $[22,25,40,41,104]$.

A recent measurement of cell water dynamics estimates the rotational correlation time for water directly interacting with biomolecular surfaces to be $27 \mathrm{ps}$ [15]. The present protein hydration water relaxation times at $300 \mathrm{~K}$ are $31 \pm 3,35 \pm 3$, and $32 \pm 6$ ps for TIP3P, TIP4P, and TIP5P, respectively. These times are very similar to each other and to the values in Ref. [15], and are approximately a factor of 4 slower then the bulk relaxation times measured in Ref. [113].

The pair angular correlations, calculated using the Kirkwood $G$-factor, indicates that TIP4P exhibits somewhat lower angular correlation, although the difference is not large $(\sim 10 \%)$. A decrease in the G-factor accompanies the dynamical transition.

Taken together, the present results suggest that the global dynamical properties of the protein and hydration water are not significantly affected by variation of the water models among TIP3P, TIP4P, and TIP5P. Although they have documented different bulk phase properties, the water models behave similarly on the protein surface for the quantities investigated.

Furthermore, it has been previously shown that the SPC/E water model [115] 
with the GROMOS protein force field [116] also reproduces the protein dynamical transition at the same temperature [96].

\section{References}

[1] JL Finney. The water molecule and its interactions: The interaction between theory, modelling, and experiment. Journal of Molecular Liquids, 90(13):303-312, 2001.

[2] M Chaplin. Do we underestimate the importance of water in cell biology? Nature Reviews Molecular Cell Biology, 7(11):861-866, NOV 2006.

[3] W Kauzmann. Some factors in the interpretation of protein denaturation. Advances in Protein Chemistry, 14:1-63, 1959.

[4] F Franks. Protein hydration. April 1988.

[5] KA Dill. Dominant forces in protein folding. Biochemistry, 29(31):71337155, AUG 71990.

[6] B Halle. Protein hydration dynamics in solution: A critical survey. Philosophical Transactions of the Royal Society B: Biological Sciences, 359:12071224, Aug 2004. 101098/rstb20041499.

[7] A Paciaroni, S Cinelli, E Cornicchi, A Francesco, and G Onori. Fast fluctuations in protein powders: The role of hydration. Chemical Physics Letters, 410(4-6):400 - 403, 2005. 
[8] M Nakasako. Water-protein interactions from high-resolution protein crystallography. Philosophical Transactions: Biological Sciences, 359(1448):1191-1206, 2004.

[9] A Oleinikova, N Smolin, I Brovchenko, A Geiger, and R Winter. Formation of spanning water networks on protein surfaces via 2D percolation transition. Journal of Physical Chemistry B, 109(5):1988-1998, 2005.

[10] N Smolin, A Oleinikova, I Brovchenko, A Geiger, and R Winter. Properties of spanning water networks at protein surfaces. Journal of Physical Chemistry B, 109(21):10995-11005, 2005.

[11] JM Zanotti, MC Bellissent-Funel, and J Parello. Hydration-coupled dynamics in proteins studied by neutron scattering and nmr: the case of the typical ef-hand calcium-binding parvalbumin. Bipphysical Journal, 76:2390-2411, May 1999.

[12] MA Williams, JM Goodfellow, and JM Thornton. Buried waters and internal cavities in monomeric proteins. Protein Science, 3(8):1224-1235, AUG 1994.

[13] VP Denisov and B Halle. Protein hydration dynamics in aqueous solution. Faraday Discussions, 103:227-244, 1996.

[14] AM Stadler, JP Embs, I Digel, GM Artmann, T Unruh, G Bueldt, and G Zaccai. Cytoplasmic water and hydration layer dynamics in human red blood 
cells. Journal of the American Chemical Society, 130:16852-16853, Nov 2008. doi: 101021/ja807691j.

[15] E Persson and B Halle. Cell water dynamics on multiple time scales. Proceedings of the National Academy of Sciences, 105(17):6266-6271, 2008.

[16] DI Svergun, S Richard, MHJ Koch, Z Sayers, S Kuprin, and G Zaccai. Protein hydration in solution: Experimental observation by $\mathrm{x}$-ray and neutron scattering. Proceedings of the National Academy of Sciences, 95(5):22672272, 1998.

[17] F Merzel and JC Smith. Is the first hydration shell of lysozyme of higher density than bulk water? Proceedings of the National Academy of Sciences, 99(8):5378-5383, 2002.

[18] F Merzel and JC Smith. High-density hydration layer of lysozymes: Molecular dynamics decomposition of solution scattering data. Journal of Chemical Information and Modeling, 45(6):1593-1599, 2005.

[19] JM Zanotti, MC Bellissent-Funel, and SH Chen. Relaxational dynamics of supercooled water in porous glass. Physical Review E, 59(3):3084-3093, Mar 1999.

[20] M-C Bellissent-Funel, SH Chen, and J-M Zanotti. Single-particle dynamics of water molecules in confined space. Physical Review E, 51(5):4558-4569, May 1995. 
[21] S Dellerue and MC Bellissent-Funel. Relaxational dynamics of water molecules at protein surface. Chemical Physics, 258(2-3):315-325, AUG 152000 .

[22] D Russo, G Hura, and T Head-Gordon. Hydration dynamics near a model protein surface. Biophysical Journal, 86(3):1852 - 1862, 2004.

[23] S Takahara, N Sumiyama, S Kittaka, T Yamaguchi, and MC Bellissent-Funel. Neutron scattering study on dynamics of water molecules in mcm-41 2determination of translational diffusion coefficient. Journal of Physical Chemistry $B, 109(22): 11231-11239,2005$.

[24] H Jansson, R Bergman, and J Swenson. Protein and solvent dynamics as studied by QENS and dielectric spectroscopy. Journal of Non-Crystalline Solids, 352(42-49):4410 - 4416, 2006.

[25] C Malardier-Jugroot, ME Johnson, RK Murarka, and T Head-Gordon. Aqueous peptides as experimental models for hydration water dynamics near protein surfaces. Physical Chemistry Chemical Physics, 10(32):4903-4908, 2008.

[26] A Froelich, F Gabel, M Jasnin, U Lehnert, D Oesterhelt, AM Stadler, M Tehei, M Weik, K Wood, and G Zaccai. From shell to cell: Neutron scattering studies of biological water dynamics and coupling to activity. Faraday Discussions, 141:117-130, 2009. 
[27] B Halle, T Andersson, S Forsen, and B Lindman. Protein hydration from water oxygen-17 magnetic relaxation. Journal of the American Chemical Society, 103:500-508, May 1981. doi: 101021/ja00393a004.

[28] CF Polnaszek and RG Bryant. Nitroxide radical induced solvent proton relaxation: Measurement of localized translational diffusion. The Journal of Chemical Physics, 81(9):4038-4045, 1984.

[29] CF Polnaszek and RG Bryant. Self-diffusion of water at the protein surface: A measurement. Journal of the American Chemical Society, 106:428-429, May 1984. doi: 101021/ja00314a032.

[30] CF Polnaszek, DA Hanggi, PW Carr, and RG Bryant. Nuclear magnetic relaxation dispersion measurement of water mobility at a silica surface. Analytica Chimica Acta, 194:311 - 315, 1987.

[31] G Carlstrom and B Halle. Water dynamics in microemulsion droplets - a nuclear-spin relaxation study. Langmuir, 4(6):1346-1352, NOV-DEC 1988.

[32] BD Armstrong and S Han. Overhauser dynamic nuclear polarization to study local water dynamics. Journal of the American Chemical Society, 131:46414647, Mar 2009. doi: 101021/ja809259q.

[33] SK Pal, J Peon, and AH Zewail. Biological water at the protein surface: Dynamical solvation probed directly with femtosecond resolution. Proceedings of the National Academy of Sciences, 99(4):1763-1768, FEB 192002. 
[34] YLA Rezus and HJ Bakker. Strong slowing down of water reorientation in mixtures of water and tetramethylurea. Journal of Physical Chemistry A, 112(11):2355-2361, 2008.

[35] SH Lee and PJ Rossky. A comparison of the structure and dynamics of liquid water at hydrophobic and hydrophilic surfaces - a molecular dynamics simulation study. The Journal of Chemical Physics, 100(4):3334-3345, 1994.

[36] AR Bizzarri and S Cannistraro. Molecular dynamics simulation evidence of anomalous diffusion of protein hydration water. Physical Review E, 53(4):R3040-R3043, Apr 1996.

[37] C Rocchi, AR Bizzarri, and S Cannistraro. Water dynamical anomalies evidenced by molecular-dynamics simulations at the solvent-protein interface. Physical Review E, 57(3):3315-3325, Mar 1998.

[38] AR Bizzarri and S Cannistraro. Molecular dynamics of water at the proteinsolvent interface. Journal of Physical Chemistry B, 106(26):6617-6633, JUL 42002.

[39] M Marchi, F Sterpone, and M Ceccarelli. Water rotational relaxation and diffusion in hydrated lysozyme. Journal of the American Chemical Society, 124(23):6787-6791, JUN 122002.

[40] D Argyris, NR Tummala, A Striolo, and DR Cole. Molecular structure and 
dynamics in thin water films at the silica and graphite surfaces. Journal of Physical Chemistry C, 112(35):13587-13599, SEP 42008.

[41] ME Johnson, C Malardier-Jugroot, RK Murarka, and T Head-Gordon. Hydration water dynamics near biological interfaces. The Journal of Physical Chemistry B, 113:4082-4092, Dec 2008. doi: 101021/jp806183v.

[42] AD MacKerell, D Bashford, M Bellott, RL Dunbrack, JD Evanseck, MJ Field, S Fischer, J Gao, H Guo, S Ha, D Joseph-McCarthy, L Kuchnir, K Kuczera, FTK Lau, C Mattos, S Michnick, T Ngo, DT Nguyen, B Prodhom, WE Reiher, B Roux, M Schlenkrich, JC Smith, R Stote, J Straub, M Watanabe, J Wiorkiewicz-Kuczera, D Yin, and M Karplus. All-atom empirical potential for molecular modeling and dynamics studies of proteins. Journal of Physical Chemistry B, 102(18):3586-3616, 1998.

[43] BR Brooks, RE Bruccoleri, BD Olafson, DJ States, S Swaminathan, and M Karplus. CHARMM - A program for macromolecular energy, minimization, and dynamics calculations. Journal of Computational Chemistry, 4:187-217, 1982.

[44] A MacKerell, J Wiorkiewicz-Kuczera, and M Karplus. An all-atom empirical energy function for the simulation of nucleic acids. Journal of the American Chemical Society, 117(48):11946-11975, 1995.

[45] DA Pearlman, DA Case, JW Caldwell, WS Ross, TE Cheatharm III, S De 
Bolt, D Ferguson, G Seibel, and P Kollmann. Amber, a package of computer programs for applying molecular mechanics, normal mode analysis, molecular dynamics and free energy calculations to simulate the structural and energetic properties of molecules. CompPhysComm, 91:1-41, 1995.

[46] WRP Scott, PH Hünenberg, IG Tironi, AE Mark, SR Billeter, J Fennen, AE Torda, T Huber, P Krüger, and WF van Gunsteren. The GROMOS biomolecular simulation program package. Journal of Physical Chemistry A, 103:3596-3607, 1999.

[47] WL Jorgensen, DS Maxwell, and J Tirado-Rives. Development and testing of the OPLS all-atom force field on conformational energetics and properties of organic liquids. Journal of the American Chemical Society, 118(45):11225$11236,1996$.

[48] B Guillot. A reappraisal of what we have learnt during three decades of computer simulations on water. Journal of Molecular Liquids, 101:219-260, 2002.

[49] MW Mahoney and WL Jorgensen. A five-site model for liquid water and the reproduction of the density anomaly by rigid, nonpolarizable potential functions. The Journal of Chemical Physics, 112:8910-8922, 2000.

[50] DR Nutt and JC Smith. Molecular dynamics simulations of proteins: Can the explicit water model be varied? Journal of Chemical Theory and Computa- 
tion, 3(4):1550-1560, 2007.

[51] WL Jorgensen, J Chandrasekhar, JD Madura, RW Impey, and ML Klein. Comparison of simple potential functions for simulating liquid water. The Journal of Chemical Physics, 79:926-935, 1983.

[52] F Parak and H Formanek. Untersuchung des Schwingungsanteils und des Kristallgitterfehleranteils des Temperaturfaktors in Myoglobin durch Vergleich von Moessbauer-absorptionsmessungen mit Roentgenstrukturdaten. Acta Crystallographica Section A, 27(6):573-578, Nov 1971.

[53] EW Knapp, SF Fischer, and F Parak. Protein dynamics from moessbauer spectra - The temperature dependence. Journal of Physical Chemistry, 86(26):5042-5047, 1982.

[54] F Parak and EW Knapp. A Consistent Picture of Protein Dynamics. Proceedings of the National Academy of Sciences, 81(22):7088-7092, 1984.

[55] W Doster, S Cusack, and W Petry. Dynamical transition of myoglobin revealed by inelastic neutron scattering. Nature, 337:754-756, Feb 1989. $101038 / 337754 a 0$.

[56] JC Smith. Protein dynamics: Comparison of simulations with inelastic neutron scattering experiments. Quarterly Reviews of Biophysics, 24(3):227291, 1991.

[57] BF Rasmussen, AM Stock, D Ringe, and GA Petsko. Crystalline ribonu- 
clease a loses function below the dynamical transition at $220 \mathrm{k}$. Nature, 357:423-424, Jun 1992.

[58] J Fitter, RE Lechner, and NA Dencher. Picosecond molecular motions in bacteriorhodopsin from neutron scattering. Biophysical Journal, 73(4):2126$2137,1997$.

[59] A Ostermann, R Waschipky, FG Parak, and GU Nienhaus. Ligand binding and conformational motions in myoglobin. Nature, 404:205-208, Mar 2000. $101038 / 35004622$.

[60] D Ringe and GA Petsko. The 'glass transition' in protein dynamics: What it is, why it occurs, and how to exploit it. Biophysical Chemistry, 105:667-680, Sep 2003.

[61] AL Tournier, J Xu, and JC Smith. Translational hydration water dynamics drives the protein glass transition. Biophysical Journal, 85(3):1871-1875, 2003.

[62] AL Tournier and JC Smith. Principal components of the protein dynamical transition. Physical Review Letters, 91(20):208106, Nov 2003.

[63] R Schulz, M Krishnan, I Daidone, and JC Smith. Instantaneous normal modes and the protein glass transition. Biophysical Journal, 96(2):476 484, 2009.

[64] AL Lee and AJ Wand. Microscopic origins of entropy, heat capacity 
and the glass transition in proteins. Nature, 411:501-504, May 2001. $101038 / 35078119$.

[65] JA Hayward and JC Smith. Temperature Dependence of Protein Dynamics: Computer Simulation Analysis of Neutron Scattering Properties. Biophysical Journal, 82(3):1216-1225, 2002.

[66] JH Roh, VN Novikov, RB Gregory, JE Curtis, Z Chowdhuri, and AP Sokolov. Onsets of anharmonicity in protein dynamics. Physical Review Letters, 95(3):038101, 2005.

[67] W Doster and M Settles. Protein-water displacement distributions. Biochimica et Biophysica Acta (BBA) - Proteins \& Proteomics, 1749:173-186, Jun 2005 .

[68] JH Roh, JE Curtis, S Azzam, VN Novikov, I Peral, Z Chowdhuri, RB Gregory, and AP Sokolov. Influence of hydration on the dynamics of lysozyme. Biophysical Journal, 91(7):2573-2588, 2006.

[69] M Krishnan, V Kurkal-Siebert, and JC Smith. Methyl group dynamics and the onset of anharmonicity in myoglobin. Journal of Physical Chemistry B, 112(17):5522-5533, 2008.

[70] IET Iben, D Braunstein, W Doster, H Frauenfelder, MK Hong, JB Johnson, S Luck, P Ormos, A Schulte, PJ Steinbach, AH Xie, and RD Young. Glassy behavior of a protein. Physical Review Letters, 62(16):1916-1919, Apr 1989. 
[71] M Ferrand, A J Dianoux, W Petry, and G Zaccai. Thermal motions and function of bacteriorhodopsin in purple membranes: effects of temperature and hydration studied by neutron scattering. Proceedings of the National Academy of Sciences, 90:9668-9672, 1993.

[72] J Fitter, OP Ernst, T Hauß, RE Lechner, KP Hofmann, and NA Dencher. Molecular motions and hydration of purple membranes and disk membranes studied by neutron scattering. European Biophysics Journal, 27:638-645, Sep 1998. 101007/s002490050175.

[73] J Fitter, SAW Verclasc, RE Lechnerc, H Seelerta, and NA Dencher. Function and picosecond dynamics of bacteriorhodopsin in purple membrane at different lipidation and hydration. FEBS Letters, 433:321-325, 1998.

[74] J Fitter. The Temperature Dependence of Internal Molecular Motions in Hydrated and Dry alpha -Amylase: The Role of Hydration Water in the Dynamical Transition of Proteins. Biophysical Journal, 76(2):1034-1042, 1999.

[75] D Vitkup, D Ringe, GA Petsko, and M Karplus. Solvent mobility and the protein 'glass' transition. Nature Structural \& Molecular Biology, 7:34-38, Jan 2000. 101038/71231.

[76] V Reat, R Dunn, M Ferrand, JL Finney, RM Daniel, and JC Smith. Solvent dependence of dynamic transitions in protein solutions. Proceedings of the National Academy of Sciences, 97(18):9961-9966, 2000. 
[77] PW Fenimore, H Frauenfelder, BH McMahon, and FG Parak. Slaving: Solvent fluctuations dominate protein dynamics and functions. Proceedings of the National Academy of Sciences, 99(25):16047-16051, 2002.

[78] A Paciaroni, S Cinelli, and G Onori. Effect of the Environment on the Protein Dynamical Transition: A Neutron Scattering Study. Biophysical Journal, 83(2):1157-1164, 2002.

[79] MF Chaplin. Water: Its importance to life. Biochemistry and Molecular Biology Education, 29:54-59, Mar 2001.

[80] L Cordone, M Ferrand, E Vitrano, and G Zaccai. Harmonic behavior of trehalose-coated carbon-monoxy-myoglobin at high temperature. Biophysical Journal, 76(2):1043-1047, 1999.

[81] AM Tsai, DA Neumann, and LN Bell. Molecular dynamics of solid-state lysozyme as affected by glycerol and water: A neutron scattering study. Biophysical Journal, 79(5):2728-2732, 2000.

[82] M Tarek and DJ Tobias. Role of protein-water hydrogen bond dynamics in the protein dynamical transition. Physical Review Letters, 88(13):138101, Mar 2002.

[83] X Ding, BF Rasmussen, GA Petsko, and D Ringe. Direct structural observation of an acyl-enzyme intermediate in the hydrolysis of an ester substrate by elastase. Biochemistry, 33(31):9285-9293, 1994. 
[84] F Parak, EN Frolov, AA Kononenko, RL Moessbauer, VI Goldanskii, and AB Rubin. Evidence for a correlation between the photoinduced electron transfer and dynamic properties of the chromatophore membranes from rhodospirillum rubrum. FEBS Letters, 117:368-372, 1980.

[85] RM Daniel, JC Smith, M Ferrand, S Hery, R Dunn, and JL Finney. Enzyme activity below the dynamical transition at $220 \mathrm{~K}$. Biophysical Journal, 75(5):2504-2507, 1998.

[86] RV Dunn, V Réat, J Finney, M Ferrand, JC Smith, and RM Daniel. Enzyme activity and dynamics: Xylanase activity in the absence of fast anharmonic dynamics. Biochemical Journal, 346:355-358, 2000.

[87] Bragger JM. Enzyme activity down to $-100^{\circ}$ C. Biochimica et Biophysica Acta (BBA)/Protein Structure and Molecular Enzymology, 1480:278-282(5), 14 July 2000.

[88] V Kurkal, RM Daniel, John L Finney, M Tehei, RV Dunn, and Jeremy C Smith. Enzyme activity and flexibility at very low hydration. Biophysical Journal, 89(2):1282-1287, 2005.

[89] E Neria, S Fischer, and M Karplus. Simulation of activation free energies in molecular systems. The Journal of Chemical Physics, 105(5):1902-1921, 1996.

[90] WE Reiher. Thesis. Harvard University, 1985. 
[91] P Mark and L Nilsson. Structure and dynamics of the TIP3P, SPC, and SPC/E water models at 298 k. Journal of Physical Chemistry A, 105(43):9954-9960, 2001.

[92] J Vojtechovsky, K Chu, J Berendzen, RM Sweet, and I Schlichting. Crystal structures of myoglobin-ligand complexes at near-atomic resolution. Biophysical Journal, 77:2153-2174, 1999.

[93] JP Ryckaert, G Ciccotti, and HJC Berendsen. Numerical integration of the cartesian equations of motion of a system with constraints: Molecular dynamics of n-alkanes. Journal of Computational Physics, 23:321-341, 1977.

[94] HJC Berendsen, JPM Postma, WF van Gunsteren, A DiNola, and JR Haak. Molecular dynamics with coupling to an external bath. The Journal of Chemical Physics, 81:3684-3690, 1984.

[95] B Hess, C Kutzner, D van der Spoel, and E Lindahl. GROMACS 4: Algorithms for highly efficient, load-balanced, and scalable molecular simulation. Journal of Chemical Theory and Computation, 4(3):435-447, MAR 2008.

[96] CF Wong, C Zheng, and JA Mccammon. Glass transition in SPC/E water and in a protein solution - A molecular-dynamics simulation study. Chemical Physics Letters, 154(2):151-154, JAN 131989.

[97] C Arcangeli, AR Bizzarri, and S Cannistraro. Role of interfacial water in the 
molecular dynamics-simulated dynamical transition of plastocyanin. Chemical Physics Letters, 291(1-2):7-14, JUL 101998.

[98] F Parak, EW Knapp, and D Kucheida. Protein dynamics - MossbauerSpectroscopy on deoxymyoglobin crystals. Journal of Molecular Biology, 161(1):177-194, 1982.

[99] M Tarek and DJ Tobias. The dynamics of protein hydration water: A quantitative comparison of molecular dynamics simulations and neutron-scattering experiments. Biophysical Journal, 79(6):3244-3257, 2000.

[100] Steinbach, PJ and Brooks, BR. Protein hydration elucidated by molecular dynamics simulation. Proceedings of the National Academy of Sciences of the United States of America, 90(19):9135-9139, oct 1993.

[101] MM Teeter, A Yamano, B Stec, and U Mohanty. On the nature of a glassy state of matter in a hydrated protein: Relation to protein function. Proc Natl Acad Sci U S A, 98(20):11242, 2001.

[102] G Caliskan, A Kisliuk, and AP Sokolov. Dynamic transition in lysozyme: Role of a solvent. Journal of Non-Crystalline Solids, 307-310:868-873, Sep 2002.

[103] D van der Spoel and PJ van Maaren. The origin of layer structure artifacts in simulations of liquid water. Journal of Chemical Theory and Computation, 2(1):1-11, 2006. 
[104] RK Murarka and T Head-Gordon. Single particle and collective hydration dynamics for hydrophobic and hydrophilic peptides. The Journal of Chemical Physics, 126(21):215101, 2007.

[105] J Rault. Origin of the Vogel-Fulcher-Tammann law in glass-forming materials: The alpha-beta bifurcation. Journal of Non-Crystalline Solids, 271(3):177-217, JUL 2000.

[106] PG Debenedetti and FH Stillinger. Supercooled liquids and the glass transition. Nature, 410:259-267, Mar 2001. 101038/35065704.

[107] W Kauzmann. The nature of the glassy state and the behavior of liquids at low temperatures. Chemical Reviews, 43(2):219-256, 1948.

[108] CA Angell. Formation of glasses from liquids and biopolymers. Science, 267(5206):1924-1935, 1995.

[109] JG Kirkwood. The dielectric polarization of polar liquids. The Journal of Chemical Physics, 7(10):911-919, 1939.

[110] S Boresch, S Ringhofer, P Hoechtl, and O Steinhauser. Towards a better description and understanding of biomolecular solvation. Biophysical Chemistry, 78(1-2):43-68, 1999.

[111] P Hoechtl, S Boresch, W Bitomsky, and O Steinhauser. Rationalization of the dielectric properties of common three-site water models in terms of their 
force field parameters. The Journal of Chemical Physics, 109(12):49274937, 1998.

[112] YLA Rezus and HJ Bakker. On the orientational relaxation of HDO in liquid water. The Journal of Chemical Physics, 123(11):114502, 2005.

[113] C Rønne, L Thrane, PO Åstrand, A Wallqvist, KV Mikkelsen, and SR Keiding. Investigation of the temperature dependence of dielectric relaxation in liquid water by thz reflection spectroscopy and molecular dynamics simulation. The Journal of Chemical Physics, 107(14):5319-5331, 1997.

[114] D Laage and JT Hynes. A molecular jump mechanism of water reorientation. Science, 311(5762):832-835, 2006.

[115] HJC Berendsen, JR Grigera, and TP Straatsma. The missing term in effective pair potentials. The Journal of Physical Chemistry, 91:6269-6271, 1987. doi: 101021/j100308a038.

[116] J Hermans, HJC Berendsen, WF van Gunsteren, and JPM Postma. A consistent empirical potential for water-protein interactions. Biopolymers, 23(8):1513-1518, 1984. 


\begin{tabular}{lrrrr}
\hline \hline & TIP3P & TIP3Pm & TIP4P & TIP5P \\
\hline$q_{\mathrm{H}} / e$ & 0.417 & 0.417 & 0.520 & 0.241 \\
$q_{\mathrm{O}} / e$ & -0.834 & -0.834 & & \\
$q_{\mathrm{M}} / e$ & & & -1.040 & \\
$q_{\mathrm{L}} / e$ & & & & -0.241 \\
$\sigma_{\mathrm{OO}} / \AA$ & 3.5364 & 3.5364 & 3.5399 & 3.5021 \\
$\epsilon_{\mathrm{O}} / \mathrm{kcal} / \mathrm{mol}$ & 0.1521 & 0.1521 & 0.1550 & 0.1600 \\
$\sigma_{\mathrm{HH}} / \AA$ & & 0.4490 & & \\
$\epsilon_{\mathrm{H}} / \mathrm{kcal} / \mathrm{mol}$ & & 0.0460 & & \\
$r_{\mathrm{OH}} / \AA$ & 0.9572 & 0.9572 & 0.9572 & 0.9572 \\
$r_{\mathrm{OM}} / \AA$ & & & 0.15 & \\
$r_{\mathrm{OL}} / \AA$ & & & & 0.7 \\
$\theta_{\mathrm{HOH}} / \mathrm{deg}$ & 104.52 & 104.52 & 104.52 & 104.52 \\
$\theta_{\mathrm{LOL}} / \mathrm{deg}$ & & & & 109.47 \\
\hline
\end{tabular}

Table 1: TIP3P, TIP4P, TIP5P, and TIP3Pm potential energy parameters

\begin{tabular}{rrcrrccccc}
\hline \hline $\mathrm{T} / \mathrm{K}$ & \multicolumn{3}{c}{ TIP3P } & \multicolumn{3}{c}{ TIP4P } & \multicolumn{3}{c}{ TIP5P } \\
\hline 150 & 0.0018 & \pm & 0.0000 & 0.0018 & \pm & 0.0002 & 0.0007 & \pm & 0.0001 \\
160 & 0.0043 & \pm & 0.0002 & 0.0016 & \pm & 0.0002 & 0.0007 & \pm & 0.0002 \\
170 & 0.0171 & \pm & 0.0007 & 0.0025 & \pm & 0.0002 & 0.0009 & \pm & 0.0001 \\
180 & 0.0444 & \pm & 0.0033 & 0.0036 & \pm & 0.0001 & 0.0009 & \pm & 0.0000 \\
190 & 0.116 & \pm & 0.0045 & 0.0086 & \pm & 0.0011 & 0.0009 & \pm & 0.0001 \\
200 & 0.3104 & \pm & 0.0075 & 0.0193 & \pm & 0.0004 & 0.0014 & \pm & 0.0000 \\
210 & 0.5058 & \pm & 0.0146 & 0.04 & \pm & 0.0007 & 0.0028 & \pm & 0.0004 \\
220 & 0.8463 & \pm & 0.0032 & 0.1306 & \pm & 0.0026 & 0.003 & \pm & 0.0001 \\
230 & 1.243 & \pm & 0.0177 & 0.2699 & \pm & 0.0010 & 0.0073 & \pm & 0.0016 \\
240 & 1.7191 & \pm & 0.0464 & 0.4948 & \pm & 0.0044 & 0.0202 & \pm & 0.0002 \\
250 & 2.3816 & \pm & 0.0269 & 0.8021 & \pm & 0.0017 & 0.0459 & \pm & 0.0072 \\
260 & 2.8328 & \pm & 0.0261 & 1.1895 & \pm & 0.0040 & 0.2601 & \pm & 0.0001 \\
280 & 3.9614 & \pm & 0.1640 & 2.1882 & \pm & 0.0674 & 1.1849 & \pm & 0.0100 \\
300 & 5.4596 & \pm & 0.0158 & 3.595 & \pm & 0.0581 & 2.8162 & \pm & 0.0190 \\
\hline
\end{tabular}

Table 2: Diffusion constants in units of $10^{-5} \mathrm{~cm}^{2} / \mathrm{s}$ calculated using the Einstein relation for TIP3P, TIP4P, and TIP5P from a $30 \AA$ cubic water box with the same electrostatic treatment as in the protein simulation. 


\begin{tabular}{lrrr}
\hline \hline & TIP3P & TIP4P & TIP5P \\
\hline$\tau_{c} / p s$ & 0.01 & 21 & 0.5 \\
$A / K$ & 3810 & 403 & 1904 \\
$T_{0} / K$ & -145 & 79 & -55 \\
\hline
\end{tabular}

Table 3: Fitted parameter values for Eq. 8

\begin{tabular}{|c|c|c|c|c|c|c|c|c|c|}
\hline & $\mathrm{T} / \mathrm{K}$ & TIP3P & TIP4P & TIP5P & & $\mathrm{T} / \mathrm{K}$ & TIP3P & TIP4P & TIP5P \\
\hline \multirow{12}{*}{$a_{0}$} & 160 & 0.08 & 0.10 & 0.09 & \multirow{12}{*}{$\tau_{0}$} & 160 & 0.00 & 1.88 & 5.11 \\
\hline & 170 & 0.08 & 0.08 & 0.11 & & 170 & 0.10 & 0.11 & 7.15 \\
\hline & 180 & 0.09 & 0.09 & 0.08 & & 180 & 0.15 & 0.18 & 0.21 \\
\hline & 190 & 0.11 & 0.10 & 0.08 & & 190 & 0.41 & 0.17 & 0.31 \\
\hline & 200 & 0.14 & 0.12 & 0.09 & & 200 & 0.83 & 0.41 & 0.29 \\
\hline & 210 & 0.16 & 0.15 & 0.12 & & 210 & 1.06 & 0.91 & 0.78 \\
\hline & 220 & 0.21 & 0.18 & 0.14 & & 220 & 1.74 & 1.26 & 0.74 \\
\hline & 230 & 0.23 & 0.19 & 0.18 & & 230 & 1.66 & 1.01 & 1.38 \\
\hline & 240 & 0.29 & 0.24 & 0.24 & & 240 & 2.18 & 1.66 & 2.06 \\
\hline & 260 & 0.40 & 0.33 & 0.25 & & 260 & 2.60 & 1.85 & 1.11 \\
\hline & 280 & 0.42 & 0.38 & 0.28 & & 280 & 1.63 & 1.39 & 0.69 \\
\hline & 300 & 0.30 & 0.32 & 0.29 & & 300 & 0.44 & 0.55 & 0.43 \\
\hline \multirow{12}{*}{$a_{1}$} & 160 & 0.06 & 0.00 & 0.00 & \multirow{12}{*}{$\tau_{1}$} & 160 & 78.22 & 1049.77 & 2710.67 \\
\hline & 170 & 0.06 & 0.05 & 0.00 & & 170 & 25.05 & 27.77 & 1984.47 \\
\hline & 180 & 0.07 & 0.08 & 0.08 & & 180 & 26.10 & 48.92 & 45.39 \\
\hline & 190 & 0.14 & 0.11 & 0.09 & & 190 & 53.70 & 41.52 & 42.55 \\
\hline & 200 & 0.20 & 0.14 & 0.12 & & 200 & 59.20 & 61.70 & 37.20 \\
\hline & 210 & 0.24 & 0.27 & 0.18 & & 210 & 56.04 & 79.61 & 63.44 \\
\hline & 220 & 0.31 & 0.30 & 0.27 & & 220 & 50.97 & 64.38 & 51.01 \\
\hline & 230 & 0.38 & 0.35 & 0.33 & & 230 & 45.34 & 44.00 & 53.98 \\
\hline & 240 & 0.41 & 0.40 & 0.39 & & 240 & 42.57 & 44.90 & 49.49 \\
\hline & 260 & 0.40 & 0.41 & 0.44 & & 260 & 38.17 & 29.87 & 24.68 \\
\hline & 280 & 0.39 & 0.44 & 0.43 & & 280 & 22.27 & 19.51 & 12.89 \\
\hline & 300 & 0.43 & 0.45 & 0.43 & & 300 & 7.32 & 10.09 & 7.46 \\
\hline \multirow{12}{*}{$1-a_{0}-a_{1}$} & 160 & 0.86 & 0.90 & 0.91 & \multirow{12}{*}{$\tau_{2}$} & 160 & 2819.49 & 3498.03 & 3679.43 \\
\hline & 170 & 0.87 & 0.87 & 0.89 & & 170 & 1714.96 & 1939.45 & 3007.36 \\
\hline & 180 & 0.84 & 0.83 & 0.84 & & 180 & 1535.37 & 1245.80 & 1650.42 \\
\hline & 190 & 0.75 & 0.79 & 0.83 & & 190 & 963.95 & 1182.51 & 1538.97 \\
\hline & 200 & 0.66 & 0.73 & 0.78 & & 200 & 890.55 & 1020.11 & 1156.62 \\
\hline & 210 & 0.60 & 0.58 & 0.69 & & 210 & 610.75 & 968.10 & 1007.51 \\
\hline & 220 & 0.48 & 0.52 & 0.59 & & 220 & 580.03 & 736.41 & 838.47 \\
\hline & 230 & 0.40 & 0.46 & 0.49 & & 230 & 594.33 & 690.84 & 741.91 \\
\hline & 240 & 0.30 & 0.35 & 0.38 & & 240 & 515.06 & 443.10 & 431.47 \\
\hline & 260 & 0.20 & 0.26 & 0.31 & & 260 & 398.39 & 349.69 & 280.67 \\
\hline & 280 & 0.19 & 0.18 & 0.29 & & 280 & 269.09 & 235.99 & 123.27 \\
\hline & 300 & 0.27 & 0.23 & 0.28 & & 300 & 86.83 & 119.99 & 86.76 \\
\hline
\end{tabular}

Table 4: Fitted parameter values for Eq. 6. $\tau_{1}, \tau_{2}$, and $\tau_{3}$ in ps. 

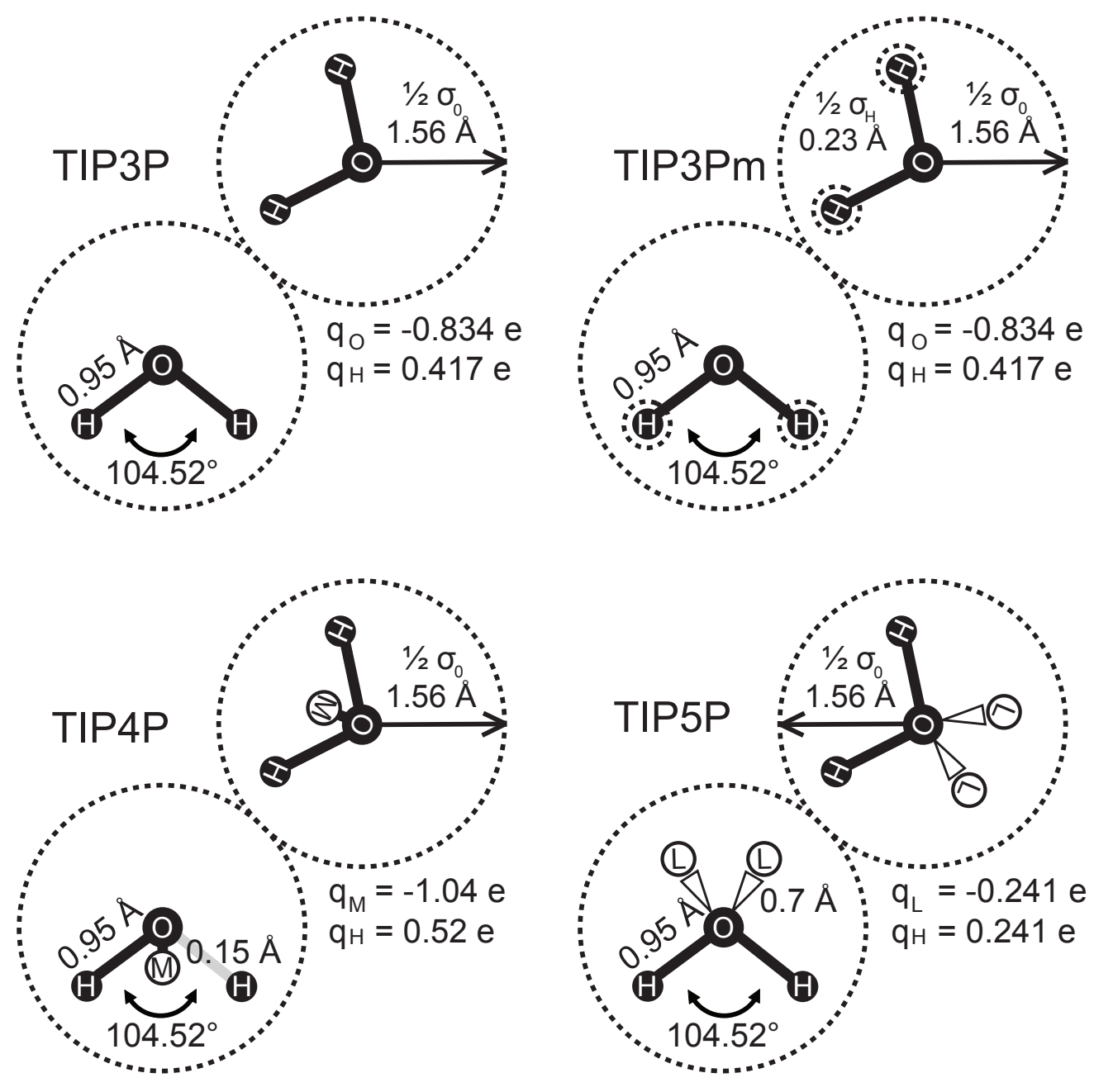

Figure 1: TIPnP geometries. Dashed lines represent $1 / 2$ of the van der Waals radius $\sigma_{0}$. 


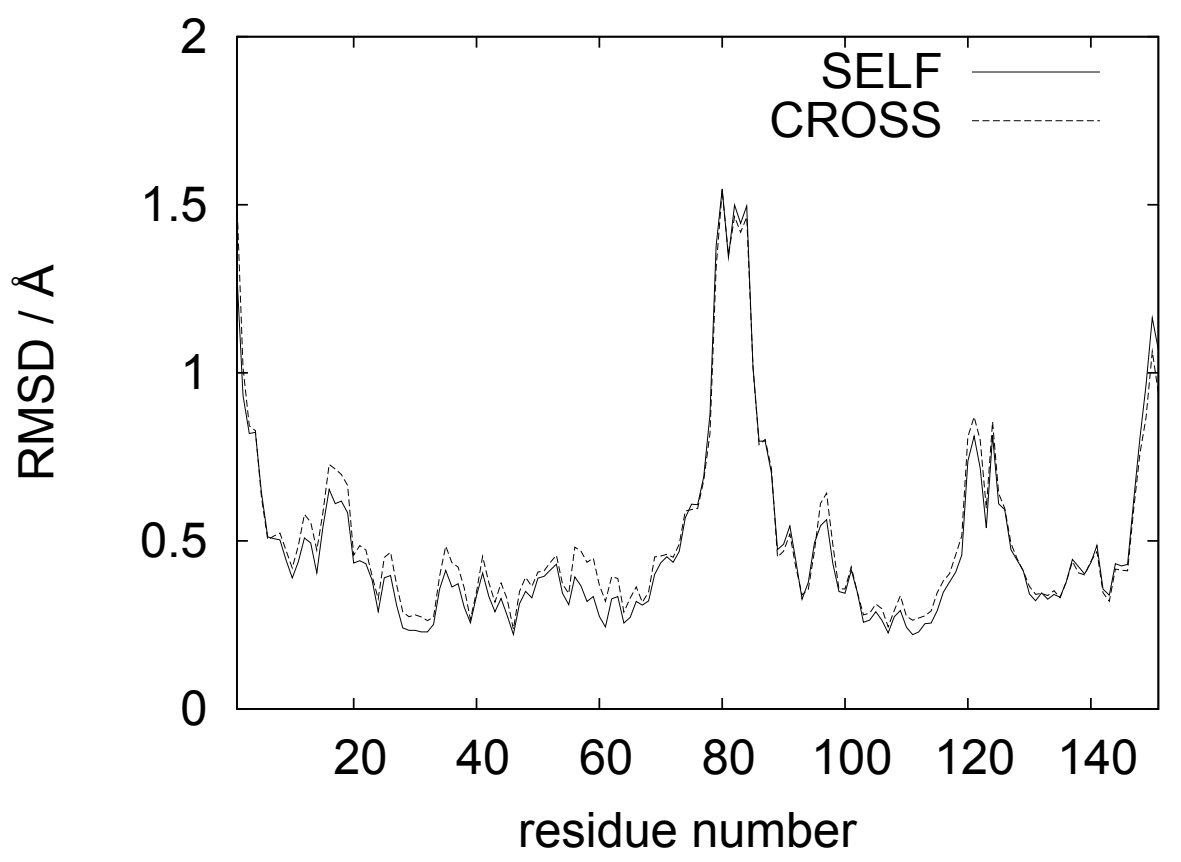

Figure 2: Backbone heavy-atom RMSD per residue at $300 \mathrm{~K}$. Average over pairs of structures solvated in the same or in different water models. "SELF" refers to RMSD values between protein structures solvated by the same water model whereas "CROSS" refers to deviations in configurations between the models. 

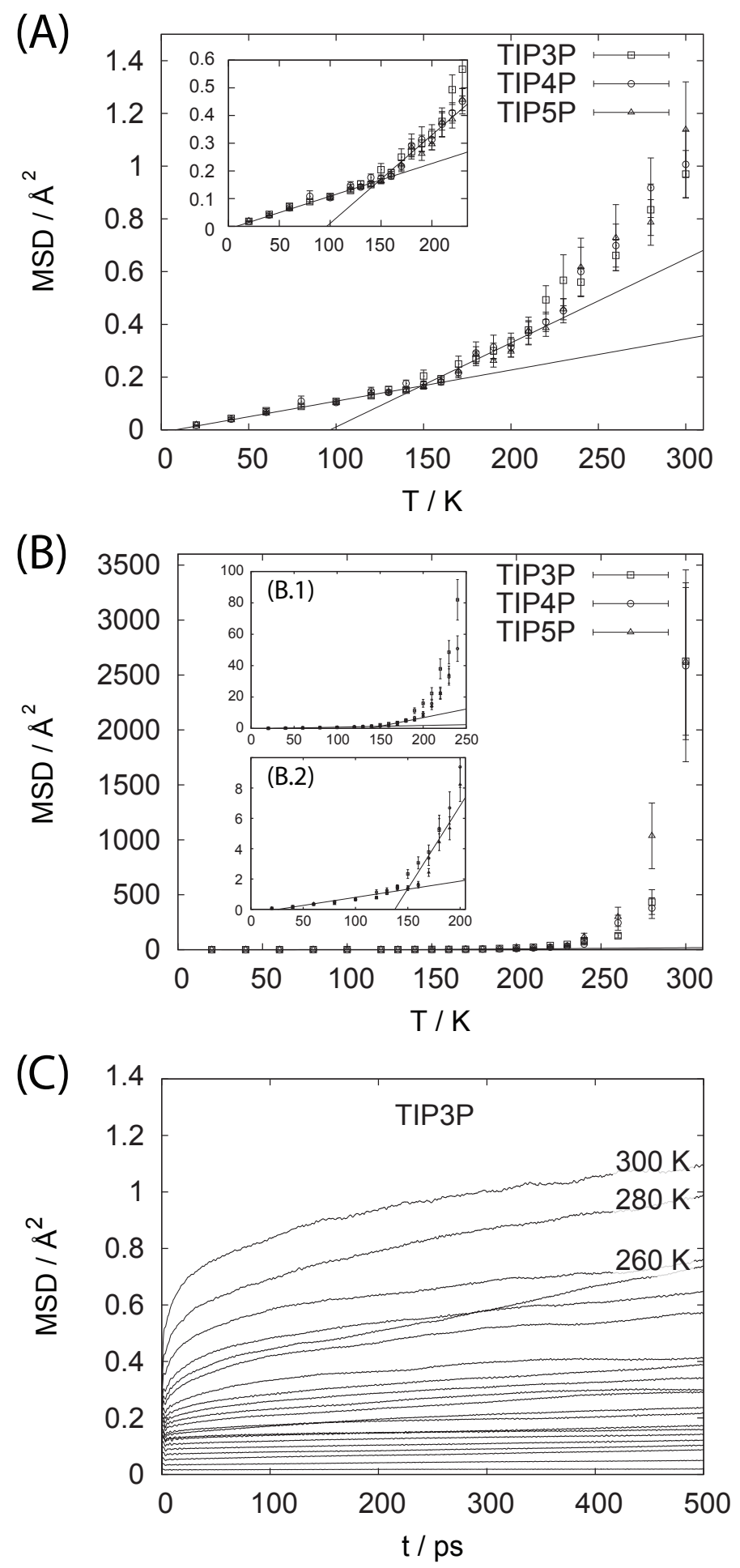

Figure 3: (A) Mean-square displacement of myoglobin solvated in TIP3P, TIP4P, or TIP5P as a function of temperature. The inset shows an expanded view of the low temperature region up to $T \approx 210 K$. (B) Meansquare displacement of the water as a function of temperature. The insets (B.1) and (B.2) give expanded views since the data cover multiple orders of magnitude. (C) Mean-square displacement of myoglobin in TIP3P as a function of time at different temperatures. 

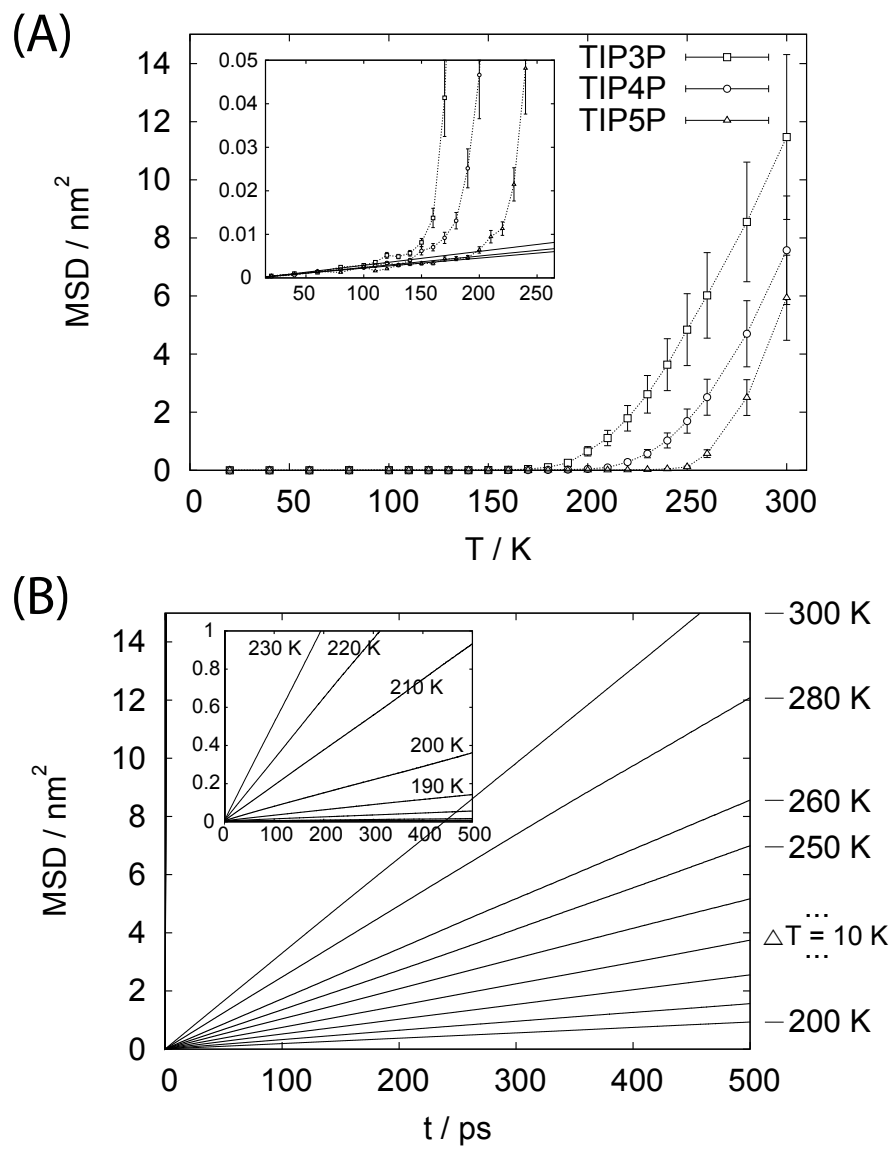

Figure 4: (A) Mean-square displacement of TIP3P, TIP4P, or TIP5P water molecules as a function of temperature from bulk water simulations. The inset shows an expanded view of the low temperature region. The solid lines corresponds to linear fits for $\mathrm{T}=20-100 \mathrm{~K}$. (B) Mean-square displacement as a function of time for TIP3P and various temperatures. The insets shows data belonging to further temperatures in an appropriate magnification of the abscissa. 


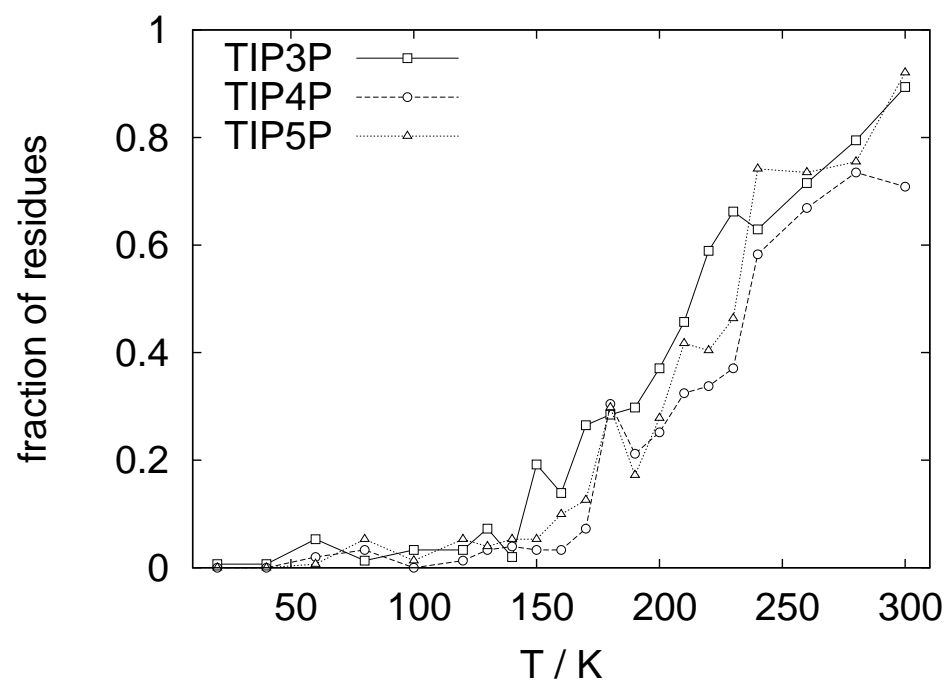

Figure 5: Fraction of residues exhibiting anharmonic dynamics. 

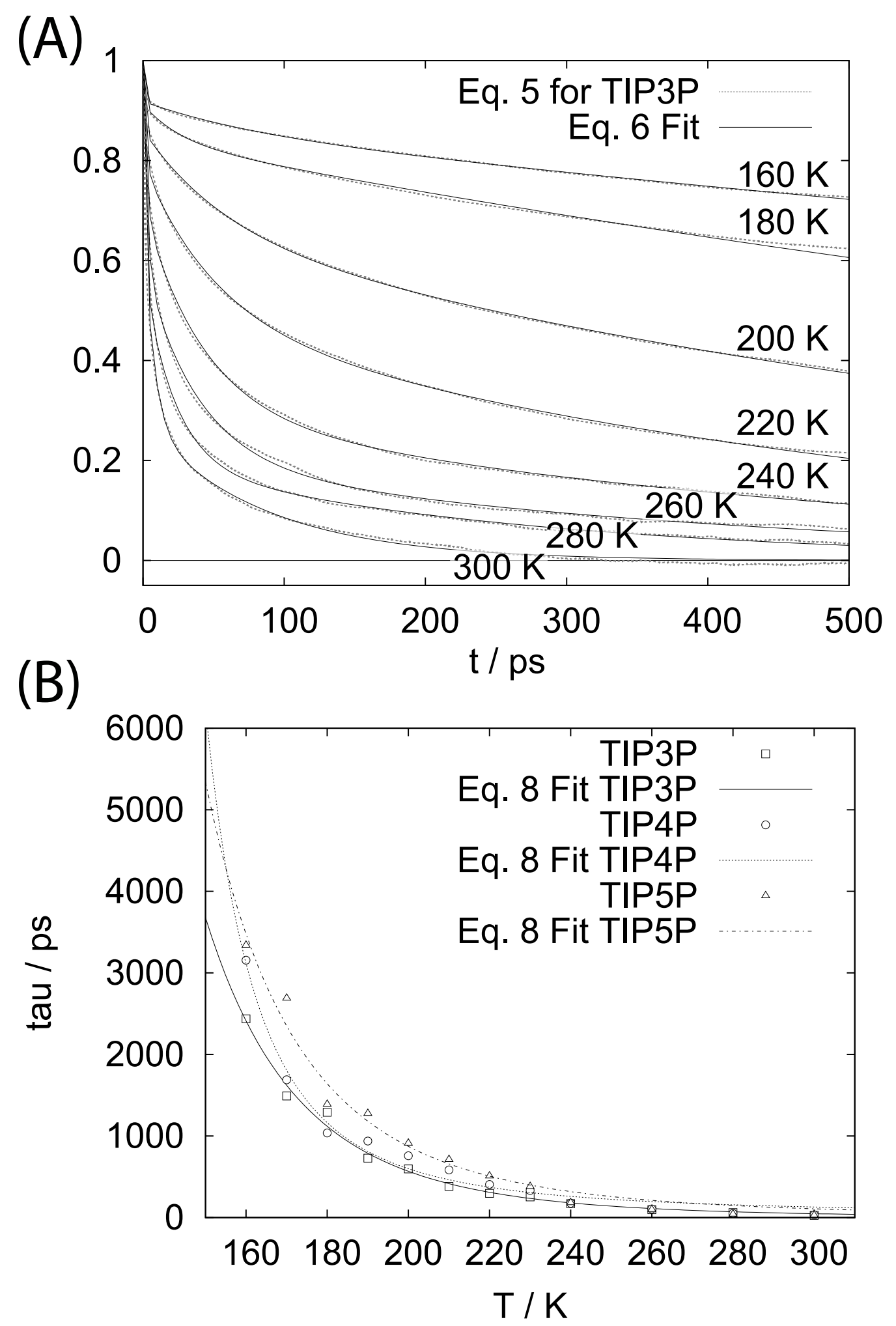

Figure 6: (A) Protein hydration water dipole orientational autocorrelation functions (Eq. 5) for TIP3P with Eq. 6 fitted. TIP4P and TIP5P data are similar. (B) Reorientational relaxation lifetimes $\tau$ fitted with the Vogel-Fulcher-Tammann equation fitted (Eq. 8) according to Eq. 7. 

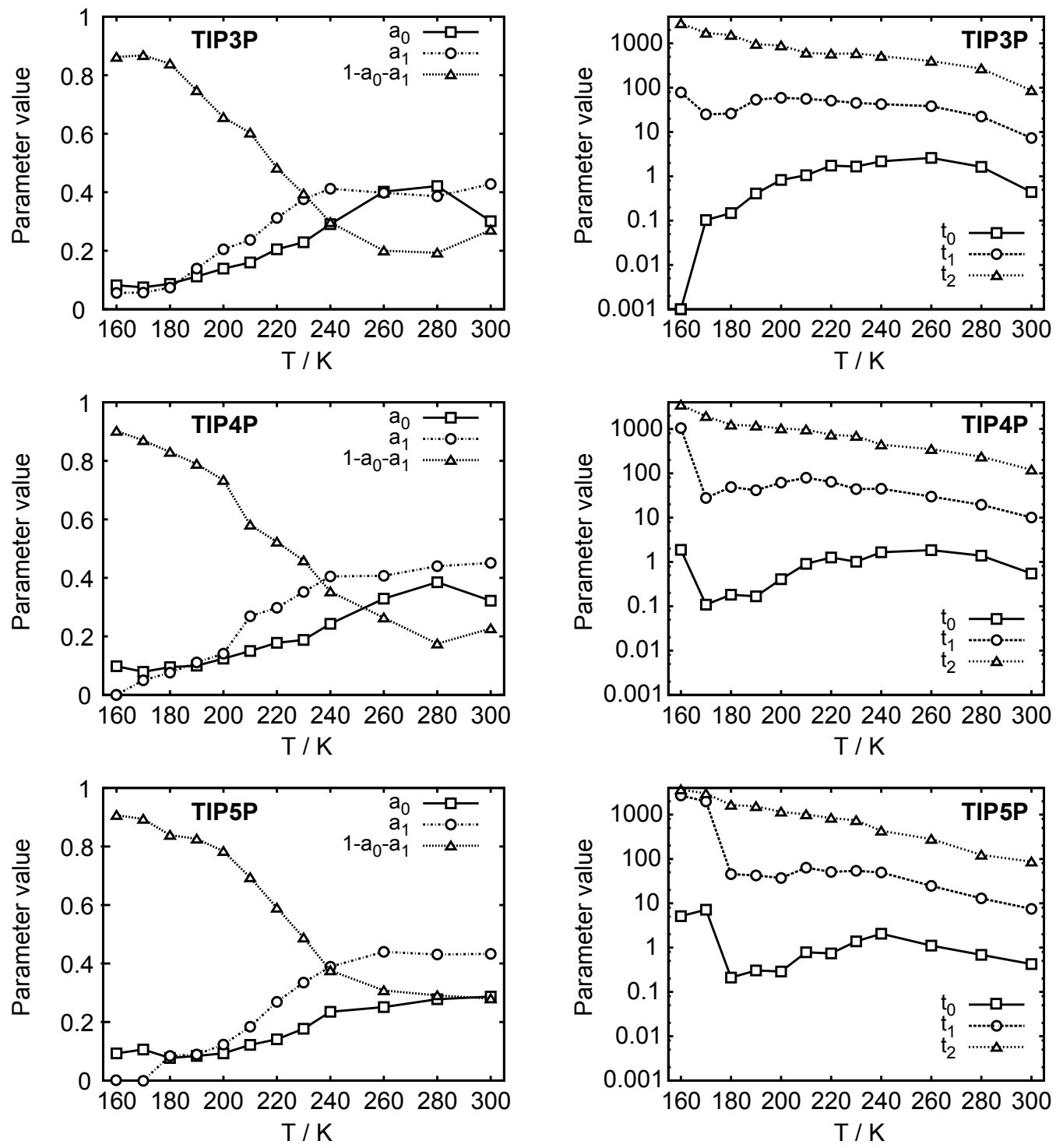

Figure 7: Fitted parameter values $a_{0}, a_{1}, 1-a_{0}-a_{1}, \tau_{0}, \tau_{1}$, and $\tau_{2}$ from Eq. 6 . Lines connect points as guide to the eye. Numerical values are given in Tbl. 4. 


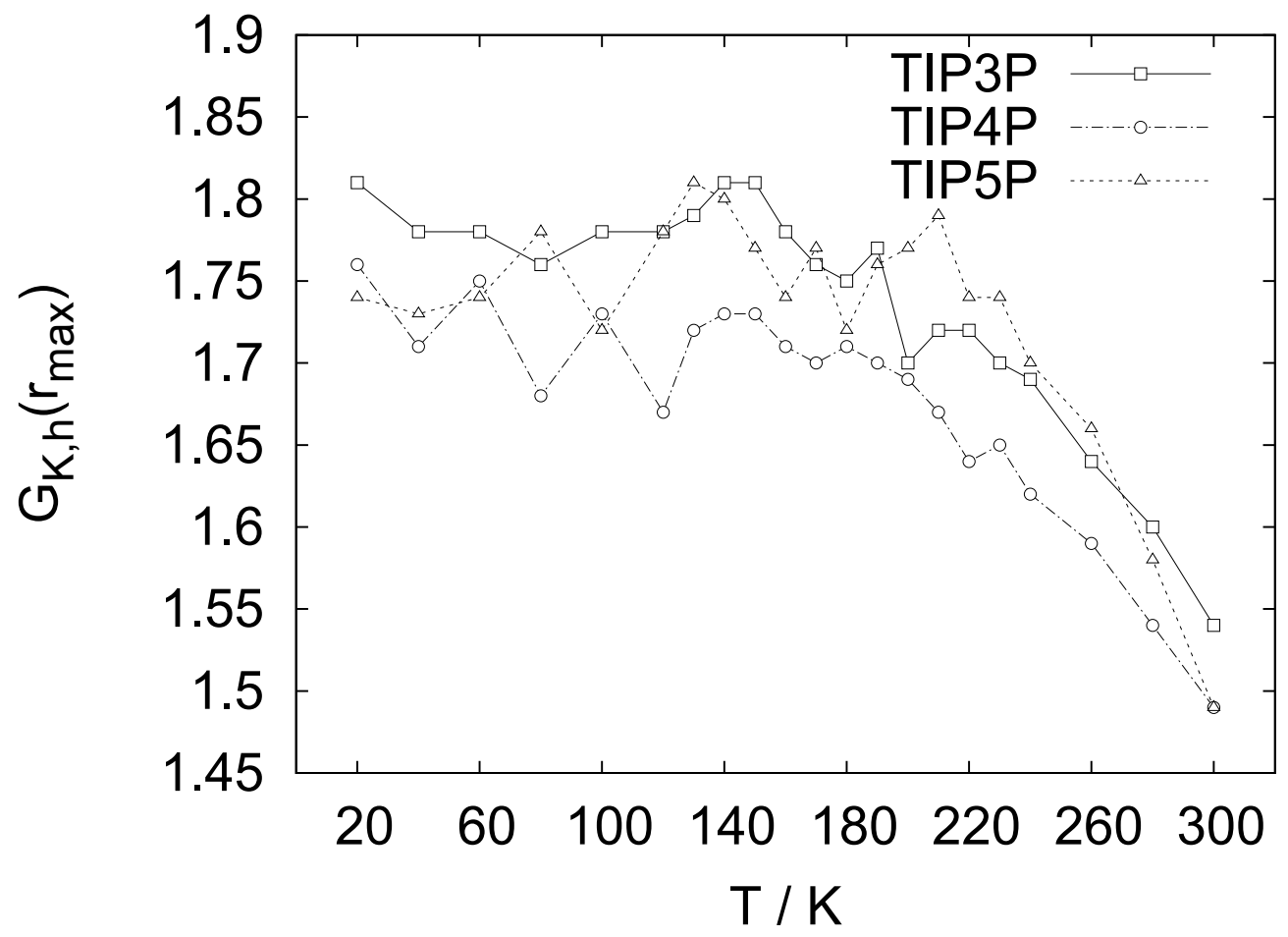

Figure 8: Local orientational ordering of water dipoles measured with the distance-dependant Kirkwood $G$ factor $G_{K, h}^{r_{\max }}(T)$ at the maximum of the oxygen-oxygen radial distribution function as a function of temperature for different water models. Lines connect points as guide to the eye. 